\title{
Research on Clinical Features of Symptomatic Epilepsy and the Risk Factors of Prognosis
}

\author{
Kang Cao, Yongquan Zhang* \\ Department of Neurology, RuiKang Hospital Affiliated to Guangxi University of Chinese Medicine, Nanning, \\ 530011, China. \\ * Corresponding Author: Yongquan Zhang
}

Keywords: Clinical features, Symptomatic epilepsy, Risk factors of prognosis

\begin{abstract}
The paper discussed the clinical characteristics and related risk factors of symptomatic epileptic seizures. A retrospective analysis of 112 cases of cerebrovascular disease confirmed by the department was carried out, including the onset time, seizure type, lesion location and prognosis. Results show that there was no significant difference between the two groups in the statistical data of sex, age, BMI, education and other general information $(\mathrm{P}>0.05)$, and the health group was significantly better than the epilepsy group in terms of annual income, quality of life score and depression grade, $\mathrm{P}<0.05$, the difference was statistically significant. After antiepileptic treatment, only 112 cases of 112 patients relapsed, 1 patient died, the treatment efficiency reached $97.32 \%$. After self-efficacy grading in 112 epilepsy patients, the difference was statistically significant $(\mathrm{P}<0.05)$ compared with the quality of life in 8 aspects. Therefore, the close attention and analysis of the clinical characteristics of the disease will help control the seizures of symptomatic epilepsy.
\end{abstract}

Epilepsy, a chronic brain disease caused by a variety of reasons, is the most common neurological disorders and disease, characterized by cerebral dysfunction caused by repeatedly excessive discharge of cerebral neurons. To attack, transient, repetitive and usually work in central nervous system can stereotype arrhythmia were characteristics. According to the recent large-scale epidemiological survey, the prevalence rate of epilepsy was 7 per thousand, the annual incidence rate of $28.2 \%$, seriously affecting people's health in China ${ }^{[1]}$. Among them, symptomatic epilepsy refers to epilepsy caused by a variety of clear or possible central nervous system lesions. It is the main group of epileptic patients. Self-efficacy refers to a person's subjective judgement or evaluation on his ability to operate in a certain field of activity ${ }^{[2]}$. At present, studies show that self-efficacy in the process of epilepsy treatment and nursing is conducive to the recovery of disease. Research on the analysis of the quality of life of patients with epilepsy and self-efficacy are less, this study analyzed the correlation between self-efficacy and the influence on the quality of life of patients with symptomatic epilepsy and related factors of quality of life, and providing a theoretical basis for the rehabilitation and quality of life in patients with symptomatic epilepsy.

\section{Data and Methods}

\subsection{General Information}

A total of 112 adults with symptomatic epilepsy who were treated in the Outpatient and Inpatient Department of Neurology Department of our hospital from January 2013 to January in -2017 were selected as the subjects. Meanwhile, 100 cases of non-epileptic patients in the physical examination center were selected as healthy control group. Epilepsy inclusion criteria: (1) after neurology physician diagnosis of adult patients with epilepsy, diagnostic evidence and / or find a variety of seizure EEG epileptiform discharge; (2) older than 18 years; (3) the duration of at least 6 months; (4) education in primary school or above, proficient in reading and understand the various scale content for survey. Exclusion criteria: (1) a serious of brain organic diseases, such as viral encephalitis and tumor patients with epilepsy; (2) chronic diseases in addition to epilepsy is suffering from other affects the quality of life of patients with epilepsy questionnaire; (3) patients with disturbance of 
consciousness or obvious mental abnormality. To understand correctly and independently check the information content of the scale; (4) to conduct neuropsychological assessment. Of all the cases, 20 cases were seizures within 2 weeks after stroke, 7 of them had epilepsy, and 10 patients had seizures more than 2 weeks after stroke. Seizure type: 14 cases of tonic clinic seizures in all patients, 11 cases of simple partial hair, 2 cases of partial seizures and 2 cases of generalized tonic clinic seizures, 2 cases of status epilepticus, and 1 cases of complex partial hair. Head CT or MRI and EEG: head CT or MRI examination confirmed 18 cases of lobar lesions located in, among them ( 7 cases in the temporal lobe, parietal lobe and occipital lobe in 4 cases, 5 cases, 2 cases of frontal lobe). There were 6 cases of subarachnoid space, 3 cases of basal ganglia, 1 cases of thalamus, 1 cases of brainstem and 1 cases of cerebellum. Electroencephalogram (EEG) was performed in 26 out of 30 cases, of which 15 cases were abnormal. Most of them were localized slow waves. Theta activity or alpha slowing down could occur on the ipsilateral lesion, or on the opposite side or bilateral sides of the lesion, and showed diffuse abnormalities. In a few cases, there is a wide range of spinous and slow complex waves or a sharp wave and a spinous wave.

\subsection{Research Methods}

The general situation of the study included age, age of onset, course of disease, educational level, family per capita income, type of attack and frequency of attack. All investigators were trained through a face-to-face questionnaire. The survey questionnaire is as follows, (1) questionnaire of quality of life in patients with epilepsy (QOLIE-31 Chinese Edition): the table set 31 questions to assess seizure worry, general quality of life, emotional health, energy / fatigue, cognitive function, social function and the influence of drugs, the overall health of a total of 8 aspects. The sum of the scores of each problem is divided by the number of problems and the initial score of each aspect. Each aspect is counted multiplied by the corresponding weight specified in the table and then added to the total score. The higher the total score, the better the quality of life. (2) self-rating Depression Scale (Self-Rating Depressing Scale, SDS): for the severity of depressive status was measured, with 20 items, divided into 4 grades, 20 items reflecting the depression state of 4 groups of specific symptoms: psychotic affective symptoms (2 items); somatic symptoms (8 items); psychomotor disorders (2); psychological disorders of depression (8 items). Each project is $1,2,3$, 4, four grades, $<50$ were divided into no depression; $<60$ were divided into mild depression; aged 60 and $<70$ were divided into moderate depression; more than 70 were divided into severe depression. (3) the self-efficacy scale of chronic disease contains 6 items: achievement experience, substitute experience, imagination experience, oral persuasion, physiological arousal and emotional state. It reflects the self-efficacy of chronic patients in many aspects. Each item adopts 1 10 level score. The higher the score is, the more confident it is. The average score of the 6 items reflects the self-efficacy level of the subjects. The reason caused by the transient cerebral hypo perfusion or insufficient energy supply in a short while the sudden loss of consciousness, mainly for disturbance of consciousness and systemic loss of muscle tone, accompanied by a lot of sweat and pale, may have severe seizures, the general continued for several seconds and a few minutes after waking, don't wake up memories, feel weakness, lethargy. Because of increased systemic vascular dilation caused by vagal reflex syncope occurred, namely vagal syncope, distributed in all age groups, common in slender preschool to adolescent girls, characterized by a loss of consciousness in a short burst, by lying, sitting or squatting suddenly appeared for the upright posture. Before the onset of often aura, such as blurred vision, dizziness, palpitation, nausea, weakness, shortness of breath, abdominal discomfort, seizures associated with autonomic symptoms, such as pale, cold sweats, generally for a few seconds gradually restored. Some patients have fatigue and lethargy after their seizures, which can last for about half an hour. It is confused with complex partial seizures, seizures and ankylosing clinic seizures, and the erect adjustment test can confirm the diagnosis. There are no epileptic discharges in EEG during attack and interictal period.

\subsection{Statistical Treatment}

The data were analyzed by SPSS 18 software, the median or the measurement data $(x+s)$ said, compared with the normal distribution of data between the two groups were $t$ test, were analyzed by 
variance analysis, normal distribution and homogeneity of variance with random design mean was compared by analysis of variance with a group of samples, completely randomized design of unequal variances by Welch-way ANONA analysis. Non-normal distribution data were ranked by rank sum test. Counting data was expressed by percentage (\%). The word 2 test was used, and Logistic regression analysis was used for multivariate analysis. The difference between $\mathrm{P}<0.05$ was statistically significant.

\section{Results}

This study investigated a total of 112 cases of adult patients with symptomatic epilepsy and 100 healthy controls, two groups of subjects in the general demographic characteristics, vital signs, years of education, height, weight, BMI index, biochemical index and other chronic disease history there were no significant differences $(\mathrm{P}>0.05)$, but the adult the symptoms of epilepsy patients than in the healthy control group annual income is low, the difference was statistically significant $(\mathrm{P}<0.05)$. Two groups of quality of life and self-rating Depression Scale score comparison, this study found that the adult symptom epilepsy group's quality of life questionnaire included 8 aspects were lower than the healthy control group, in the middle and lower levels, the difference was statistically significant $(\mathrm{P}<0.01)$. Among the adult symptomatic epileptic patients, the score of anxiety was the lowest, followed by a comprehensive quality of life score, and the overall health score was the highest. In the self-rating depression scale, the overall score of adults with symptomatic epilepsy is moderate depression, and the total score of healthy control group is no depression, and the score difference is statistically significant $(\mathrm{P}<0.01)$.

Table 1 research object general information contrast

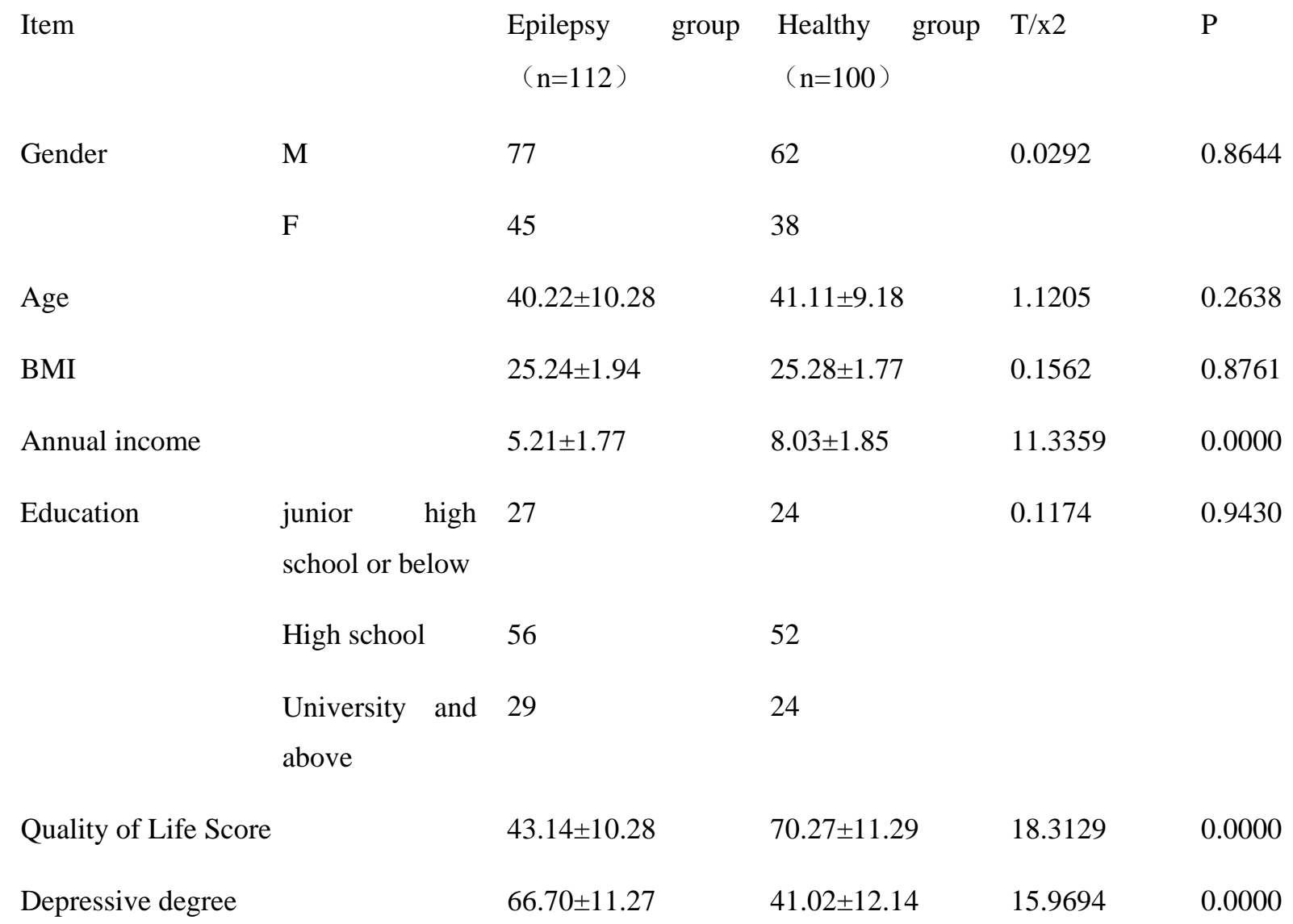

All cases were treated with antiepileptic drugs in addition to the treatment of the original disease during the treatment. 37cases (33.04\%) did not use antiepileptic drugs, 60 cases were controlled by single drug, accounting for $53.57 \%, 15$ cases were $13.39 \%$ or more than 2 drugs. For this group of patients can better control the seizures without the need for long-term medication in 90 cases 
(80.36\%); another 11 patients (9.82\%) need long-term use of antiepileptic drugs, medication during the seizure of the 11 cases $(9.82 \%)$, still attack in 2 cases; 1 cases died of status epilepticus treatment, the total efficiency of $97.32 \%$.

Before a migraine with aura, such as visual hallucinations, abnormal sensation and mild change of consciousness, often in the duration of 4 minutes to 1 hours, the content of visual hallucination is relatively simple, such as simple highlights, dark spots, fuzzy, ictal headache, lasting 2 to 4 hours, the incidence of children or interictal often accompanied by abdominal pain. Consciousness is light, or only a reduced level of consciousness, short duration, strong stimulation cannot wake up, and self-injury caused by falling. After the attack, there is vomiting and somnolence, and I see the symptoms of ophthalmoplegia and hemiplegia. In addition, metabolic diseases such as hypoglycemia, hypocalcemia, periodic paralysis, chronic heavy metal poisoning, renal encephalopathy can cause epileptic seizures, even if there is a genuine seizure occurs repeatedly, is not necessarily epilepsy.

To adult patients with symptomatic epilepsy self-efficacy analysis, self-efficacy score ranged from 1 to 10 , the object of study self-efficacy is divided into 3 levels, 1 to 3 points for the junior level, 4 to 7 points for the intermediate level, 8 to 10 points for the senior level, group of adult patients with symptomatic epilepsy were for $(6.70+1.27)$ points. The low level accounted for $6.25 \%(7 / 112)$, the intermediate level accounted for $75.89 \%$ (85/112), the advanced level accounted for $17.86 \%(20 / 112)$, $93.75 \%$ of the patients were above the average level, and $6.25 \%$ of the patients were at the low level.

We introduce self-efficacy and adult epilepsy patients with epilepsy patients' life quality questionnaire in 8 aspects (the concerns of the onset, general quality of life, emotional health, energy / fatigue, cognitive function, drug effects, social function and general health) Estimation module analysis of curve estimation, we found the correlation of self-efficacy and to attack worry, the comprehensive quality of life, emotional health, drug effects and general health, finally had statistical significance in the quality of life score $(\mathrm{P}<0.05)$.

Table 2 different levels of self-efficacy epilepsy patients quality of life score comparison

\begin{tabular}{|c|c|c|c|c|c|c|c|c|}
\hline Grouping & Onset & $\begin{array}{l}\text { overall } \\
\text { quality of } \\
\text { life }\end{array}$ & $\begin{array}{l}\text { Emotional } \\
\text { well-being, }\end{array}$ & $\begin{array}{l}\text { energy } \\
\text { / } \\
\text { fatigue }\end{array}$ & $\begin{array}{l}\text { cognitive } \\
\text { function }\end{array}$ & $\begin{array}{l}\text { drug } \\
\text { effects }\end{array}$ & $\begin{array}{l}\text { social } \\
\text { functioning }\end{array}$ & $\begin{array}{l}\text { general } \\
\text { health }\end{array}$ \\
\hline $\begin{array}{c}1-3 \\
(n=7)\end{array}$ & $\begin{array}{r}66.13 \\
\pm 14.25\end{array}$ & $60.13 \pm 5.28$ & 1.27 & $\begin{array}{l}72.16 \pm \\
11.67\end{array}$ & 68 & $\begin{array}{l}66.13 \\
\pm 5.38\end{array}$ & $.12 \pm 6.18$ & 7.17 \\
\hline $\begin{array}{c}4-7 \\
(n=85)\end{array}$ & $\begin{aligned} & 50.24 \\
\pm & 10.92\end{aligned}$ & $52.25 \pm 5.30$ & $64.27 \pm 10.66$ & $\begin{array}{l}60.27 \pm \\
11.62\end{array}$ & $50.21 \pm 7.51$ & $\begin{array}{l}49.23 \\
\pm 4.28\end{array}$ & $55.12 \pm 7.77$ & $59.13 \pm 8.99$ \\
\hline $\begin{array}{c}8-10 \\
(n=20)\end{array}$ & $\begin{aligned} & 41.24 \\
\pm & 6.38\end{aligned}$ & $6.89^{44.24 \pm}$ & $50.24 \pm 8.71$ & $\begin{array}{l}51.26 \pm \\
8.88\end{array}$ & $40.26 \pm 7.33$ & $\begin{array}{l}38.27 \\
\pm 5.13\end{array}$ & $40.23 \pm 11.26$ & $44.21 \pm 6.13$ \\
\hline $\mathrm{P}$ & $<0.05$ & $<0.05$ & $<0.05$ & $<0.05$ & $<0.05$ & $<0.05$ & $<0.05$ & $<0.05$ \\
\hline
\end{tabular}

\section{Conclusions}

Symptomatic epilepsy is the main group in epilepsy. The analysis of multiple epileptic causes at home and abroad shows that symptomatic epilepsy is the first place in different age groups. Usually in clinical recurrent seizures caused great physical and psychological pain to patients, but also seriously affected the patient's education, employment, marriage and family, even if the seizure free patients, in daily life, learning and employment and work long suffered frustration will also have an adverse effect on the patient's life quality ${ }^{[3]}$. Due to various physiological and psychological factors and social cognition, resulting in adverse effects on physical, cognitive, psychological and social function of the patients with epilepsy patients, self-assessment is reduced, thereby reducing the value of self-positioning, thereby affecting their own potential, can aggravate the degree of seizures, severely affected patients and their families the quality of life ${ }^{[4]}$.

This research adopts the research at home and abroad are that the quality of life of patients with epilepsy were recognized as validity and reliability were higher (QOLIE-31 Chinese version) was used to investigate the quality of life of adult patients with symptomatic epilepsy, found that patients quality of life scores were significantly decreased than that of healthy controls, the differences were statistically differences between the 8 aspects, which is the lowest score the onset of concern, the 
domestic and foreign research shows that it is difficult to control the seizures and unpredictable can seriously affect the quality of life of patients, and the higher the frequency of seizures, seizure patients worry is more obviously, unpredictable attacks give patients and their families to bring different degree of psychological burden. Besides, fear of being discriminated against by others can further aggravate the psychological pressure of patients, lose confidence in treatment and get rid of the society. This is the social problem that medical workers should pay attention to. On the comprehensive quality of life score after the attack of the concerns, epilepsy is a chronic disease, such as poor control, can cause irreversible damage to brain function, and long-term use of antiepileptic drugs may also cause certain effects on brain function, causing cognitive function and social function of patients is poor, these factors can affect adult patients the quality of life of symptomatic epilepsy. Therefore, although effective control of seizures is the root of improving the quality of life of epileptic patients, we should educate patients' knowledge about diseases, so that patients can have a comprehensive understanding of epilepsy and understand the effect and side effects of drug therapy.

The author also conducted a self-rating Depression Scale score of patients with symptomatic epilepsy, with previous reports, the symptoms of epilepsy patients score higher than that of normal controls, the overall moderate depression, recurrent seizures bring heavy psychological pressure and social pressure to the patients, can lead to depression. Can also aggravate epilepsy and depression, leading to the quality of life of patients with repeated vicious spiral further decline. Therefore, we should pay attention to the depressive state of the patients with adult symptomatic epilepsy and examine the early stage of the disease to improve the rate of diagnosis and treatment. Isolated first attack or acute seizure control, do not recommend long-term use of antiepileptic drugs; stroke 2-3 month after the conventional treatment of epilepsy, epilepsy recommendations for long-term drug treatment; status epilepticus after stroke, according to status epilepticus treatment. The positive control of epileptic seizures can improve the quality of life of the patients.

\section{References}

[1] Hao Meimei, Qin Na, Zhang Yan, et al. Clinical characteristics and risk factors of prognosis: 216 cases of symptomatic epilepsy [J]. Chinese Journal of Neurosurgical Disease Research, 2016, 15(4): 301-304.

[2] Lu Yingyu, Qin Xinghua, Huang Bingwen. Evaluation Analysis of Quality of Life and Self-efficacy in Patients with Adult Symptomatic Epilepsy [J]. Medical Innovation of China, 2015, 12(32): 8-11.

[3] Liu Fengying, Cai Xuekun, Zhao Dan. Clinical significance of changes on serum humoral immune in newly diagnosed adult patients with non-symptomatic epilepsy [J]. Chinese Journal of Practical Nervous Diseases, 2014, 17(22): 1-2.

[4] Xu Yuansheng. Clinical analysis of 98 patients with symptomatic epilepsy secondary to cerebrovascular disease [J]. Chinese Journal of New Clinical Medicine, 2011, 4(6):542-544. 